\title{
29
}

\section{Adaptive control of semilinear stochastic evolution equations}

\author{
L. Stettner \\ Institute of Mathematics Polish Academy of Sciences \\ Sniadeckich 8, 00-950 Warsaw, Poland
}

\begin{abstract}
In the paper a nearly self optimal strategy is constructed for the control problem of stochastic semilinear evolution equation depending on an unknown parameter with path wise average per unit time cost functional.
\end{abstract}

\section{Keywords}

Stochastic evolution equations, adaptive control, self-optimality

\section{INTRODUCTION}

Let $\left(H,|\cdot|_{H}\right)$ be a separable Hilbert space and $\left(\Omega, F, F_{t}, P^{0}\right)$ be a probability space. Consider the following semilinear stochastic evolution equation on $H$

$d X=(A X+F(X)) d t+B(X) d W \quad X(0)=x \in H$

where $A$ is a generator of a $C_{0}$ semigroup $S(t)$ on $H$ and $W(t)$ is a cylindrical Wiener process on $H$ adapted to $F_{t}$.

Assume

(A1) $\exists L>0$ such that $|F(z)-F(y)|_{H} \leq|z-y|_{H}$ and $\|B(z)-B(y)\|_{L(H)} \leq|z-y|_{H}$, for $z, y \in H$

(A2) $\sup _{z \in H}\left\|B^{-1}(z)\right\|_{L(H)}<\infty$

(A3) $\exists \beta \in(0,1)$ such that for each $T>0, \quad \int_{0}^{T}\left(1+t^{-\beta}\right)\|S(t)\|_{2}^{2} d t<\infty$

It follows from Da Prato and Zabczyk (1992) that under (A1)-(A3) there exists a unique mild solution $X(t)$ to the equation (1) i.e. an $H$ valued process $X(t)$ such that for $t \geq 0$

$X(t)=S(t) x+\int_{0}^{t} S(t-s) F(X(s)) d s+\int_{0}^{t} S(t-s) B(X(s)) d W(s)$

Moreover $X(t)$ has a version with continuous trajectories that is a Markov process on $H$ with transition operator $P_{t}^{0}$ and (see Peszat and Zabczyk (1994)) 
(a1) $\exists c_{t}$ that is bounded for $t$ from compact subsets of $(0, \infty)$ such that $\mid P_{t}^{0} \psi(x)-$ $P_{t}^{0} \psi(y)\left|\leq c_{t}\right| x-\left.y\right|_{H} \sup _{z \in H}|\psi(z)|$, for $x, y \in H$ and bounded Borel measurable functions $\psi$ on $H$,

(a2) $X$ is irreducible i.e. $P_{t}^{0}(x, \mathcal{O})>0$ for $x \in H, t>0$, and open set $\mathcal{O}$

Let $U$ and $A$ be given compact metric spaces of control and unknown parameters resp., and let $D: H \times U \times A \mapsto H$ and $c: H \times U \mapsto R$. Furthermore assume

(A4) the mappings $D$ and $c$ are bounded and continuous in $v \in U$, and $D$ is moreover Lipschitz in $\alpha$, uniformly with respect to other parameters,

Denote by $\mathcal{A}$ the class of all Borel measurable functions from $H$ in $U$. Given $\alpha^{0} \in A$ and $u \in \mathcal{A}$, by an infinite dimensional version of Girsanov theorem (see Duncan Pasik-Duncan and Stettner (1995)) we can define a new measure $P^{\alpha^{0}, u}$ on $\Omega$, such that the restrictions $P_{\mid t}^{\alpha^{0}, u}$ and $P_{\mid t}^{0}$ of $P^{\alpha^{0}, u}$ and $P^{0}$ resp., to $\sigma$-field $F_{t}$ satisfy $P_{\mid t}^{\alpha^{0}, u}(d \omega)=\exp \left[\zeta^{\alpha^{0}, u}(x, t)\right] P_{\mid t}^{0}(d \omega)$ with

$$
\begin{aligned}
\zeta^{\alpha^{0}, u}(x, t)=\int_{0}^{t} & <B^{-1}(X(s)) D\left(X(s), u(X(s)), \alpha^{0}\right), d W(s)> \\
& -\frac{1}{2} \int_{0}^{t}\left|B^{-1}(X(s)) D\left(X(s), u(X(s)), \alpha^{0}\right)\right|_{H}^{2} d s
\end{aligned}
$$

and where $X(0)=x$. Under the measure $P^{\alpha^{0}, u}$, the process $X(t)$ becomes a solution to the following integral equation

$$
\begin{array}{r}
X(t)=S(t) x+\int_{0}^{t} S(t-s)\left(F(X(s))+D\left(X(s), u(X(s)), \alpha^{0}\right)\right) d s \\
+\int_{0}^{t} S(t-s) B(X(s)) d W(s)
\end{array}
$$

and the solution to (4) in the above weak sense is unique. In what follows we shall study the case when the state process $X(t)$ is a solution to (4) and $\alpha^{0}$ is unknown. Our aim is to construct a control $v_{t}=u_{t}(X(t))$, where $u_{t} \in \mathcal{A}$ such that under measure $P^{\alpha^{0}, u}$ the path wise cost functional

$$
J\left(\left(v_{t}\right)\right)=\limsup _{t \rightarrow \infty} t^{-1} \int_{0}^{t} c\left(X(s), v_{s}\right) d s
$$

is minimal. The study of the cost functional (5) requires certain ergodic properties of the controlled process $X(t)$. For this purpose we shall impose the following assumption

(A5) there exists a Markov time $\tau$ such that for $\tau_{n+1}=\tau_{n}+\tau \circ \theta_{\tau_{n}},\left(\theta_{t}\right.$ is a Markov shift operator corresponding to $X(t))$, the process $X\left(\tau_{n}\right)$ is Markov on a compact set $\Gamma$ and uniformly ergodic which means that $\forall \alpha \in A, u \in \mathcal{A} \quad \exists 0<\gamma<1 \exists \eta_{u}^{\alpha} \in \mathcal{P}(\Gamma)$ such that

$$
\sup _{u \in \mathcal{A}} \sup _{\alpha \in A} \sup _{x \in \Gamma} \sup _{B \in \mathcal{B}(\Gamma)}\left|P_{x}^{\alpha, u}\left\{X\left(\tau_{n}\right) \in B\right\}-\eta_{u}^{\alpha}(B)\right| \leq \gamma^{n}
$$


Moreover we have $\sup _{u \in \mathcal{A}} \sup _{\alpha \in A} \sup _{x \in \Gamma} E_{x}^{\alpha, u}\left\{\tau^{2}\right\}<\infty, \sup _{x \in \Gamma} E_{x}^{0}\left\{\tau^{2}\right\}<\infty$, $\inf _{u \in \mathcal{A}} \inf _{\alpha \in A} \inf _{x \in \Gamma} E_{x}^{\alpha, u}\left\{\tau^{2}\right\}>0$ and $E_{x}^{\alpha, u}\left\{T_{\Gamma}\right\}<\infty$, for $x \in H, u \in \mathcal{A}, \alpha \in A$, with $T_{B}=\inf \{s \geq 0, X(s) \in B\}$ for $B \in \mathcal{B}(H)$.

For the model we study the assumption (A5) is not very restrictive. In section 3 a particular form of Markov time $\tau$ and set $\Gamma$ will be shown. It is known (see e.g. Duncan Pasik-Duncan and Stettner (1994b)) that under (A5) the optimal value of cost functional (5) is $P^{\alpha^{0}, u}$ a.e. equal to that of

$\bar{J}_{x}^{\alpha^{0}}\left(\left(v_{t}\right)\right)=\limsup _{t \rightarrow \infty} t^{-1} E_{x}^{\alpha^{0}}\left\{\int_{0}^{t} c\left(X(s), v_{s}\right) d s\right\}$

We shall also assume

(A6) (Roxin condition) for each $x \in H$ the set $\left\{\left(\begin{array}{l}D(x, v) \\ c(x, v)\end{array}\right), v \in U\right\}$ is convex in the Hilbert space $H \times R$.

which is a technical assumption that can be removed by considering a larger class of so called relaxed controls (see e.g. Gatarek and Sobczyk (1994)).

Adaptive control of LQ infinite dimensional models has been studied in Duncan Goldys and Pasik-Duncan (1991), Duncan Maslowski Pasik-Duncan (1994), (1995), and Aihara (1995). In the paper adaptive control of semilinear stochastic evolution equations is considered. The adaptive procedure is an infinite dimensional adaptation of Duncan PasikDuncan and Stettner (1994b). Results of the paper can in particular be applied to a system of controlled reaction diffusion equations (see e.g. Duncan Pasik-Duncan and Stettner (1995)).

\section{CONTINUITY PROPERTIES}

In this section we list a family of consequences of the assumptions (A1)-(A6), which will be used in the proof of the main Theorem in section 6 .

Notice first that by (a1) and (a2) the measures $P_{t}^{0}(x, \cdot)$, for $x \in H$ and $t>0$ are equivalent. Fix $\bar{x} \in H$ and $\bar{t}>0$ and let $\mu=P_{\bar{t}}^{0}(\bar{x}, \cdot)$. By Theorem 1 of Duncan PasikDuncan and Stettner (1995) the measures $\mu$ and $P_{t}^{\alpha, u}(x, \cdot)$, for $x \in H$ and $u \in \mathcal{A}$ are equivalent. Moreover we have

Lemma 1 Under (A1)-(A6) the measures

$\pi_{u}^{\alpha}(B)=\frac{\int_{\Gamma} E_{x}^{\alpha, u}\left\{\int_{0}^{\tau} \chi_{B}(X(s)) d s\right\} \eta_{u}^{\alpha}(d x)}{\int_{\Gamma} E_{x}^{\alpha, u}\{\tau\} \eta_{u}^{\alpha}(d x)}$

for $B \in \mathcal{B}(H)$, are unique invariant for the transition semigroup $P_{t}^{\alpha, u}$ and the family $\left\{\pi_{u}^{\alpha}, \alpha \in A, u \in \mathcal{A}\right\}$ is tight.

Proof. One can easily check that the measures $\pi_{u}^{\alpha}$ are invariant for the semigroup $P_{t}^{\alpha, u}$. Since transition operators $P_{t}^{\alpha, u}(x, \cdot)$ are equivalent for $x \in H$ and $t>0$, the invariant 
measures $\pi_{u}^{\alpha}$ are unique. It remains to show the tightness of the family $\left\{\pi_{u}^{\alpha}, \alpha \in A, u \in \mathcal{A}\right\}$. For this purpose we shall prove that the family of measures $\Psi_{x}^{\alpha, u}(B)=\frac{E_{x}^{\alpha, u}\left(\int_{0}^{\tau} \chi_{B}(X(s)) d s\right\}}{E_{x}^{\alpha, u}\{\tau\}}$, $x \in \Gamma, \alpha \in A, u \in \mathcal{A}$ is tight. In fact, for given sequences $x_{n} \in \Gamma, \alpha_{n} \in A, u_{n} \in \mathcal{A}$, by compactness of $\Gamma, A$ and Lemma 2 of Duncan Pasik-Duncan and Stettner (1995) one can find subsequences, for simplicity denoted by $n$ and $x \in \Gamma, \alpha \in A, u \in \mathcal{A}$ such that $x_{n} \rightarrow x$, $\alpha_{n} \rightarrow \alpha$ and $D\left(x, u_{n}(x), \alpha_{n}\right) \rightarrow D(x, u(x), \alpha)$ in the weak * topology of $L_{\infty}(H, \eta, H)$. Mimicking the proof of the first part of the Theorem 2 of Duncan Pasik-Duncan and Stettner (1995) for each $t>0$ and $\phi \in C(H, R)$ we have

$\int_{\Omega} \int_{0}^{\tau \wedge t} \phi(X(s)) d s \quad \exp \left[\zeta^{\alpha_{n}, u_{n}}\left(x_{n}, t\right)\right] d P^{0} \rightarrow \int_{\Omega} \int_{0}^{\tau \wedge t} \phi(X(s)) d s \quad \exp \left[\zeta^{\alpha, u}\left(x_{n}, t\right)\right] d P^{0}$

Since by (A5), $\sup _{u \in \mathcal{A}} \sup _{\alpha \in A} \sup _{x \in \Gamma} E_{x}^{\alpha, u}\left\{\tau^{2}\right\}<\infty$, for any $\phi \in C(H, R)$

$E_{x_{n}}^{\alpha_{n}, u_{n}}\left\{\int_{0}^{\tau} \phi(X(s)) d s\right\} \rightarrow E_{x}^{\alpha, u}\left\{\int_{0}^{\tau} \phi(X(s)) d s\right\}$

and consequently $\Psi_{x_{n}}^{\alpha_{n}, u_{n}}(\phi) \rightarrow \Psi_{x}^{\alpha, u}(\phi)$, which completes the proof of the tightness of $\pi_{u}^{\alpha}$ with $\alpha \in A, u \in \mathcal{A}$.

By Remark 6 of Duncan Pasik-Duncan and Stettner (1995) and Lemma 1 we immediately obtain

Corollary 1 Under (A1)-(A6) for each $\alpha \in A$ there exists an optimal control function $u^{\alpha} \in \mathcal{A}$ for the cost functional $\bar{J}_{x}^{\alpha}((u(X(t))))$.

Let $\rho_{A}$ denote a metric on $A$. From an infinite dimensional version of Proposition 2.2 and (19) of Duncan Pasik-Duncan and Stettner (1994a), using Lipschitian of $D$ in $\alpha$ we obtain

Lemma 2 Under (A1)-(A5) for each $\epsilon>0$ there exists $\delta>0$ such that if $\rho_{A}\left(\alpha, \alpha^{\prime}\right)<\delta$ we have

$\sup _{u \in \mathcal{A}} \sup _{x \in \Gamma} \sup _{B \in \mathcal{B}(\Gamma)}\left|P_{x}^{\alpha, u}\{X(\tau) \in B\}-P_{x}^{\alpha^{\prime}, u}\{X(\tau) \in B\}\right|<\epsilon$

and

$\sup _{u \in \mathcal{A}} \sup _{x \in \Gamma} \sup _{B \in \mathcal{B}(H)}\left|E_{x}^{\alpha, u}\left\{\int_{0}^{\tau} \chi_{B}(X(s)) d s\right\}-E_{x}^{\alpha^{\prime}, u}\left\{\int_{0}^{\tau} \chi_{B}(X(s)) d s\right\}\right|<\epsilon$

Denote by $\|\eta\|_{\text {var }}$ the variation norm of the measure $\eta$. By the proof of Proposition 1 (in particular (18)) of Stettner (1993) we have

Lemma 3 Under (A5) there exist constants $K_{1}$ and $\dot{K_{2}}$ such that for $\alpha, \alpha^{\prime} \in A$

$\sup _{u \in \mathcal{A}}\left\|\eta_{u}^{\alpha}-\eta_{u}^{\alpha^{\prime}}\right\|_{\text {var }} \leq K_{1} K_{2} \sup _{u \in \mathcal{A}} \sup _{x \in \Gamma} \sup _{B \in \mathcal{B}(\Gamma)}\left|P_{x}^{\alpha, u}\{X(\tau) \in B\}-P_{x}^{\alpha^{\prime}, u}\{X(\tau) \in B\}\right|$ 
Combining (8) and (11), (12), (13) we obtain

Corollary 2 Under (A1)-(A5) the mapping $A \ni \alpha \mapsto \pi_{u}^{\alpha}$ is uniformly in $u \in \mathcal{A}$ continuous in variation norm of $\mathcal{P}(H)$.

Consequently by Corollary 3 and Lemma 2 of Stettner (1993) we obtain

Corollary 3 Under (A1)-(A5) for every $\epsilon>0$, there exists a finite class $\mathcal{A}(\epsilon)=$ $\left\{u_{1}, \ldots, u_{r(\epsilon)}\right\}$ of $\epsilon$ - optimal control functions for the cost functionals $\bar{J}_{x}^{\alpha}$ with $\alpha \in A$ i.e.

$\forall \alpha \in A \quad \exists i \in\{1, \ldots, r(\epsilon)\} \quad \bar{J}_{x}^{\alpha}\left(\left(u_{i}(X(t))\right)\right) \leq \inf _{u \in \mathcal{A}} \bar{J}_{x}^{\alpha}((u(X(t))))+\epsilon$

For $\alpha, \alpha^{\prime} \in A$ and $u \in \mathcal{A}$ define an information measure $K_{u}\left(\alpha, \alpha^{\prime}\right)$ as follows

$K_{u}\left(\alpha, \alpha^{\prime}\right)=\int_{H}\left|B^{-1}(z)\left(D(z, u(z), \alpha)-D\left(z, u(z), \alpha^{\prime}\right)\right)\right|_{H}^{2} \pi_{u}^{\alpha^{\prime}}(d z)$

It has the following important properties

Lemma 4 Under (A1)-(A5) for every $u \in \mathcal{A}$ the mapping $A \times A \ni\left(\alpha, \alpha^{\prime}\right) \mapsto K_{u}\left(\alpha, \alpha^{\prime}\right)$ is continuous. Moreover if $K_{u}\left(\alpha, \alpha^{\prime}\right)=0$ we have $\pi_{u}^{\alpha}=\pi_{u}^{\alpha^{\prime}}$.

Proof. The continuity follows from Corollary 2 and Lipschitian in $\alpha$ of $D$. If $K_{u}\left(\alpha, \alpha^{\prime}\right)=0$, by equivalence of measures $\pi_{u}^{\alpha}$ and $\mu, D(x, u(x), \alpha)=D\left(x, u(x), \alpha^{\prime}\right)$ for $\mu$ almost all $x \in H$. Therefore by the first part of the proof of Theorem 2 of Duncan Pasik-Duncan and Stettner $(1995), P_{x}^{\alpha, u}(x, \cdot)=P_{x}^{\alpha^{\prime}, u}(x, \cdot)$ for $x \in H$, and consequently $\pi_{u}^{\alpha}=\pi_{u}^{\alpha^{\prime}}$.

Let $p_{m}$ be projection operators on $\mathcal{D}\left(A^{*}\right)$, the domain of the adjoint operator to $A$ such that for $x \in H,\left|x-p_{m} x\right|_{H} \rightarrow 0$ as $m \rightarrow \infty$. By analogy to (15) define

$K_{u}^{m}\left(\alpha, \alpha^{\prime}\right):=\int_{H}\left|p_{m} B^{-1}(z)\left(D(z, u(z), \alpha)-D\left(z, u(z), \alpha^{\prime}\right)\right)\right|_{H}^{2} \pi_{u}^{\alpha^{\prime}}(d z)$

Lemma 5 Under (A1)-(A5) for every $u \in \mathcal{A}$ we have

$\sup _{\alpha, \alpha^{\prime} \in A}\left|K_{u}^{m}\left(\alpha, \alpha^{\prime}\right)-K_{u}\left(\alpha, \alpha^{\prime}\right)\right| \rightarrow 0$

as $m \rightarrow \infty$.

Proof. The proof is based on the Lipschitzianity in $\alpha$ of $D$, boundedness of $B^{-1}(x)$ (by (A2)) and Corollary 2.

\section{CONSTRUCTION OF THE MARKOV TIME $\tau$}

This section is devoted to the construction of a particular Markov time $\tau$, for which assumption (A5) is satisfied. Recall first Lemma 5 of Da Prato Gatarek Zabczyk (1992) 
Lemma 6 Under (A1)-(A4) for $p>\frac{2}{\beta} \quad \exists C>0$ such that $\forall R>0$

$$
P_{1}^{\alpha, u}(x, K(R, \beta)) \geq 1-C R^{-1}\left(1+|x|_{H}^{p}\right) \quad P_{1}^{0}(x, K(R, \beta)) \geq 1-C R^{-1}\left(1+|x|_{H}^{p}\right)
$$

provided $|x|_{H}^{p} \leq R$, where $K(R, \beta)$ is a compact set in $H$ given by the formula

$K(R, \beta)=\left\{x \in H: x=S(1) z+G_{1} g(1)+G_{2^{-1} \beta} h(1),|z|_{H}^{p} \leq R,|g|_{L_{p}}^{p} \leq R,|h|_{L_{p}}^{p} \leq R\right\}(19)$

with $G_{\beta^{\prime}} f(1)=\int_{0}^{1}(1-s)^{\beta^{\prime}-1} S(1-s) f(s) d s$ for $\beta^{\prime} \in(0,1]$ and any bounded Borel measurable $f:[0,1] \mapsto H$.

Let $B(r)=\left\{x \in H,|x|_{H}<r\right\}, \quad \bar{B}(r)=\left\{x \in H,|x|_{H} \leq r\right\}$. Assume

(B1) $\exists r_{0}$ such that for $r_{1}>r_{0}$ we have $\sup _{|x|_{H}=r_{1}} \sup _{u \in \mathcal{A}} \sup _{\alpha \in A} E_{x}^{\alpha, u}\left\{T_{\bar{B}\left(r_{0}\right)}^{4}\right\}<\infty \quad$ and $\sup _{|x|_{H}=r_{1}} E_{x}^{0}\left\{T_{\bar{B}\left(r_{0}\right)}^{4}\right\}<\infty$.

For given $r_{0}$ such that (B1) holds we choose $R$ such that $C R^{-1}\left(1+r_{0}^{p}\right) \leq \theta<1$ with $p>\frac{2}{\beta}$. Then we find $r_{1}>2 r_{0}$ such that $K(R, \beta) \subset B\left(2^{-1} r_{1}\right)$ and for some $\kappa>0 \sup _{|x|_{H} \leq r_{0}} \sup _{u \in \mathcal{A}} \sup _{\alpha \in A} P_{x}^{\alpha, u}\left\{\exists t \in[0,1], X(t) \in H \backslash B\left(r_{1}\right)\right\} \leq 1-\kappa$ and $\sup _{|x|_{H} \leq r_{0}} P_{x}^{0}\left\{\exists t \in[0,1], X(t) \in H \backslash B\left(r_{1}\right)\right\} \leq 1-\kappa$. Let now $\Gamma=K(R, \beta)$ and

$$
\tau=T_{H \backslash B\left(r_{1}\right)}+\sigma \circ \theta_{T_{H \backslash B\left(r_{1}\right)}} \quad \sigma=\inf \left\{s+1: s \geq 0, X(s) \in \bar{B}\left(r_{0}\right), X(s+1) \in \Gamma\right\}
$$

We have

Lemma 7 Under (A1)-(A4) and (B1) we have

$\sup _{u \in \mathcal{A}} \sup _{\alpha \in A} \sup _{x \in \Gamma} E_{x}^{\alpha, u}\left\{\tau^{2}\right\}<\infty \quad \sup _{x \in \Gamma} E_{x}^{0}\left\{\tau^{2}\right\}<\infty$

Proof. Let $\bar{\tau}=T_{H \backslash B\left(r_{1}\right)}+T_{\bar{B}\left(r_{0}\right)} \circ \theta_{T_{H \backslash B\left(r_{1}\right)}}$, and $\bar{\tau}_{1}=\tau, \bar{\tau}_{n+1}=\bar{\tau}_{n}+\bar{\tau} \circ \theta_{\bar{\tau}_{n}}$, for $n=1,2, \ldots$ Define $s(0)=\inf \left\{j \geq 1: \bar{\tau}_{j} \geq 1\right\}$ and for $i \geq 1, s(i)=\inf \left\{j \geq i: \bar{\tau}_{j} \geq \bar{\tau}_{i}+1\right\}$. Let $\tilde{\tau}=\inf \left\{\bar{\tau}_{s^{n}(0)}+1, X\left(\tilde{\tau}_{s^{n}(0)}+1\right) \in \Gamma, n=0,1, \ldots\right\}$ with $s^{n+1}(0)=s\left(s^{n}(0)\right)$ and $\tau_{s^{0}(0)}:=0$. If $|X(0)|_{H} \leq r_{0}$, we have $\tau \leq \tilde{\tau}$. Since by the proof of Lemma 2.3 of Maslowski and Seidler (1993), for any $r>0$, $\sup _{|x|_{H} \leq r} \sup _{u \in \mathcal{A}} \sup _{\alpha \in A} E_{x}^{\alpha, u}\left\{T_{H \backslash B(r)}^{4}\right\}<\infty \quad$ and $\sup _{|x|_{H} \leq r} E_{x}^{0}\left\{T_{H \backslash B(r)}^{4}\right\}<\infty$, and $\theta, \kappa<1$, it can be shown (the details are left to the reader) that

$\sup _{u \in \mathcal{A}} \sup _{\alpha \in A} \sup _{x \in \Gamma} E_{x}^{\alpha, u}\left\{\tilde{\tau}^{2}\right\}<\infty, \quad \sup _{x \in \Gamma} E_{x}^{0}\left\{\tilde{\tau}^{2}\right\}<\infty$

from which the assertion of lemma follows.

Let $\tau_{n}$ be defined as in (A5) with $\tau$ given in (20). To complete the proof of (A5) we need to show (6). We have 
Proposition 1 Under (A1)-(A4) and (B1), the process $X\left(\tau_{n}\right)$ is uniformly ergodic i.e. (6) of (A5) holds.

Proof. By the proof of Theorem 2.1 of Duncan Pasik-Duncan and Stettner (1994a) it suffices to show that for $\delta>0$

$$
\sup _{x, y \in \Gamma} \sup _{u \in \mathcal{A}} \sup _{\alpha \in A} \sup _{B \in \mathcal{B}(H)}\left|P_{x}^{\alpha, u}\{X(\tau) \in B\}-P_{y}^{\alpha, u}\{X(\tau) \in B\}\right| \leq 1-\delta
$$

Suppose (23) does not hold i.e. for some $x_{n}, y_{n} \in \Gamma, u_{n} \in \mathcal{A}, \alpha_{n} \in A$ and $B_{n} \in \mathcal{B}(H)$ we have $P_{x_{n}}^{\alpha_{n}, u_{n}}\left\{X(\tau) \in B_{n}\right\} \rightarrow 1$ and $P_{y_{n}}^{\alpha_{n}, u_{n}}\left\{X(\tau) \in B_{n}\right\} \rightarrow 0$ as $n \rightarrow \infty$. Without loss of generality we can assume that $x_{n} \rightarrow \bar{x}$ and $y_{n} \rightarrow \bar{y}$. By Girsanov theorem and finiteness of the second moment of $\tau$, we obtain $P_{y_{n}}^{0}\left\{X(\tau) \in B_{n}\right\} \rightarrow 0$ and consequently from (a1), $P_{\bar{y}}^{0}\left\{X(\tau) \in B_{n}\right\} \rightarrow 0$. Using the equivalence of transition probabilities of $X\left(\tau_{n}\right)$ we have $P_{\bar{x}}^{0}\left\{X(\tau) \in B_{n}\right\} \rightarrow 0$ and then by (a1) again, $P_{x_{n}}^{0}\left\{X(\tau) \in B_{n}\right\} \rightarrow 0$. The last convergence by Girsanov theorem implies $P_{x_{n}}^{\alpha_{n}, u_{n}}\left\{X(\tau) \in B_{n}\right\} \rightarrow 0$, a contradiction.

\section{ESTIMATION AND CONTROL}

To make MLE procedure feasible we add to the ML estimator the projection operator $p_{m}$ and additionally assume

(B2) for each $x \in H, B^{-1}(x): \mathcal{D}\left(A^{*}\right) \mapsto \mathcal{D}\left(A^{*}\right)$

Then we estimate the unknown parameter $\alpha^{0}$ maximizing over $\alpha \in A$ value of

$$
\begin{array}{r}
L_{m}^{t}(\alpha):=\int_{0}^{t}<p_{m} B^{-1}(X(s)) D(X(s), u(X(s)), \alpha), p_{m} B^{-1}(X(s))[d X(s) \\
-(A X(s)+F(X(s))) d s]>-2^{-1} \int_{0}^{t}\left|p_{m} B^{-1}(X(s)) D(X(s), u(X(s)), \alpha)\right|_{H}^{2} d s
\end{array}
$$

which is equivalent to the maximization of

$$
\begin{aligned}
\int_{0}^{t} & <p_{m} B^{-1}(X(s))\left(D(X(s), u(X(s)), \alpha)-D\left(X(s), u(X(s)), \alpha^{0}\right), p_{m} d W(s)>\right. \\
& -2^{-1} \int_{0}^{t}\left|p_{m} B^{-1}(X(s))\left(D(X(s), u(X(s)), \alpha)-D\left(X(s), u(X(s)), \alpha^{0}\right)\right)\right|_{H}^{2} d s
\end{aligned}
$$

To construct a nearly optimal adaptive strategy we fix $\epsilon>0$. By Corollary 3 we can find a finite class of $\epsilon$ optimal control functions $\mathcal{A}(\epsilon)=\left\{u_{1}, \ldots, u_{r(\epsilon)}\right\}$. Let

$T(\epsilon):=\left\{a_{i} \in R^{+}, a_{i}+r \leq a_{i+1}, a_{0}=0\right.$ such that $\frac{\epsilon}{2 r\|c\|} \leq \liminf _{n \rightarrow \infty} n_{1} \sum_{i=0}^{n-1} \chi_{T(\epsilon)}(i)$ and $\left.\lim \sup _{n \rightarrow \infty} n_{1} \sum_{i=0}^{n-1} \chi_{T(\epsilon)}(i) \leq \frac{\epsilon}{r\|c\|}\right\}$,

with $\|c\|$ standing for supremum norm, and $r:=r(\epsilon)$. We have the following

Lemma 8 Under (A1)-(A5) for any $\epsilon^{\prime}>0$ there exists a $\delta>0$ and a positive integer $m$ such that $\sup _{\alpha, \alpha^{\prime} \in A}\left|K_{u}^{m}\left(\alpha, \alpha^{\prime}\right)-K\left(\alpha, \alpha^{\prime}\right)\right| \leq \frac{\delta}{6}$, and for any $\{0,1\}$ valued sequence $\beta^{i}(k)$, 
$i=1,2, \ldots, k \in\{1, \ldots, r\}$ which for each $i$ it takes value 1 only at one $k \in\{1, \ldots, r\}$, and $\beta^{i}(k)=1$ for $i=a_{j}+k-1, a_{j} \in T(\epsilon)$, we have for $\alpha, \alpha^{\prime} \in A$

if $\liminf _{n \rightarrow \infty} n^{-1} \sum_{i=0}^{n-1} \sum_{k=1}^{r} \beta^{i}(k) K_{u_{k}}^{m}\left(\alpha, \alpha^{\prime}\right)<\delta$ then $\sup _{u \in \mathcal{A}(\epsilon)}\left\|\pi_{u}^{\alpha}-\pi_{u}^{\alpha^{\prime}}\right\|_{v a r}<\epsilon^{\prime}$.

Proof. It follows from Lemma 5, Lemma 4 and the definition of the set $T(\epsilon)$.

We continue our construction. Take $\delta$ and $m$ such that Lemma 8 holds with $\epsilon^{\prime} \leq \frac{\epsilon}{\|c\|}$. By Corollary 2 and there exists a finite set $A(\delta) \subset A$ such that for any $\alpha \in A$ one can find $\bar{\alpha} \in A(\delta)$ for which $\sup _{u \in \mathcal{A}}\left\|\pi_{u}^{\alpha}-\pi_{u}^{\bar{\alpha}}\right\|_{v a r} \leq \frac{\epsilon}{\|c\|}$ and $\sup _{u \in \mathcal{A}(\epsilon)} K_{u}^{m}(\bar{\alpha}, \alpha) \leq \frac{\delta}{3}$.

Then we choose a positive integer $N$ such that (its existence follows from the uniform ergodicity of $\left.X\left(\tau_{n}\right)\right)$ for all $x \in \Gamma, u \in \mathcal{A}(\epsilon), \alpha \in A(\delta), \alpha^{\prime} \in A$ we have

$$
\begin{array}{r}
\mid E_{x}^{\alpha^{\prime}, u}\left\{\int_{0}^{\tau_{N}}\left|p_{m} B^{-1}(X(s))\left(D(X(s), u(X(s)), \alpha)-D\left(X(s), u(X(s)), \alpha^{\prime}\right)\right)\right|_{H}^{2} d s\right\} \\
\left(E_{x}^{\alpha^{\prime}, u}\left\{\tau_{N}\right\}\right)^{-1}-K_{u}^{m}\left(\alpha, \alpha^{\prime}\right) \mid \leq \frac{\delta}{3}
\end{array}
$$

and

$\left|E_{x}^{\alpha^{\prime}, u}\left\{\int_{0}^{\tau_{N}} c(X(s), u(X(s))) d s\right\}\left(E_{x}^{\alpha^{\prime}, u}\left\{\tau_{N}\right\}\right)^{-1}-\int_{E} c(z, u(z)) \pi_{u}^{\alpha}(d z)\right| \leq \epsilon$.

For a given $\epsilon>0$ we have just constructed $\mathcal{A}(\epsilon), T(\epsilon), \delta, m, N, A(\delta)$. We are now in position to define an adaptive control strategy $\left(\hat{v}_{t}\right)$, which can be described as follows.

We wait until the process $X(s)$ enters the set $\Gamma$. Then we start our procedure. At moments $\tau_{N i}, i=1, \ldots$ we choose control functions $u$ from the set $\mathcal{A}(\epsilon)$ accordingly to the following algorithm

- if $i=a_{r}+k-1$, with $a_{r} \in T(\epsilon)$ and $k \in\{1, \ldots, r\}$ we use the function $u_{k}$ for the next $\tau_{N}$ units of time,

- otherwise we maximize $L_{m}^{\tau_{N i}}(\alpha)$ over $\alpha \in A(\delta)$ i.e. determine the value $\hat{\alpha}_{N i}:=$ $\operatorname{argmax}_{\alpha \in A(\delta)}\left\{L_{m}^{\tau_{N i}}(\alpha)\right\}$ and use for the next $\tau_{N}$ units of time the control function $u$ from $\mathcal{A}(\epsilon)$ that is $\epsilon$ optimal for the parameter $\hat{\alpha}_{N i}$

In other words the strategy is to force all control functions from $\mathcal{A}(\epsilon)$ in rare moments of time (determined by $T(\epsilon)$ ) while in the other moments use control functions that are $\epsilon$ optimal for the current value of the estimation over the finite set $A(\delta)$. An alternative to the forced use all control functions from $\mathcal{A}(\epsilon)$ is a randomization as in Duncan PasikDuncan and Stettner (1994b).

Theorem 1 Under (A1)-(A6) and (B2) or (A1)-(A4), (A6), (B1), (B2) we have $J\left(\left(\hat{v}_{t}\right) \leq\right.$ $\inf _{u \in \mathcal{A}} \vec{J}_{x}^{\alpha^{0}}\left((u(X(t)))+5 \epsilon \quad P_{x}^{\alpha^{0}}\right.$ a.e.

Proof. We follow the consideration of the proof of Theorem 1 of Duncan Pasik-Duncan and Stettner (1994b). Notice that if $\alpha \in A(\delta)$ is a frequent point of the estimation, we have $\liminf _{n \rightarrow \infty} n^{-1} \sum_{i=0}^{n-1} \sum_{k=1}^{r} \beta^{i}(k) K_{u_{k}}^{m}\left(\alpha, \alpha^{0}\right)<\delta$ and consequently by Lemma 8 we have $\sup _{u \in \mathcal{A}(\epsilon)}\left\|\pi_{u}^{\alpha}-\pi_{u}^{\alpha^{0}}\right\|_{\text {var }}<\frac{\epsilon}{\|c\|}$. The details are left to the reader. 


\section{REFERENCES}

Aihara, S.I. (1995) On adaptive boundary control for stochastic parabolic systems with unknown potential coefficient. IEEE Trans. Aut. Cont., submitted.

Da Prato, G., Gatarek, D. and Zabczyk, J. (1992) Invariant measures for semilinear stochastic equations. Stoch. Anal. Appl., 10, 387-408.

Da Prato, G. and Zabczyk, J. (1992) Stochastic equations in infinite dimensions. Cambridge University Press.

Duncan, T.E., Goldys, B. and Pasik-Duncan, B. (1991) Adaptive Control of linear stochastic evolution systems. Stochastics \& Stochastics Rep., 36, 71-90.

Duncan, T.E., Maslowski, B. and Pasik-Duncan, B. (1994) Adaptive boundary and point control of linear stochastic distributed parameter systems. SIAM J. Control Optimiz., 32, 648-672.

Duncan, T.E., Maslowski, B. and Pasik-Duncan, B. (1995) Adaptive boundary control of linear stochastic distributed parameter systems described by analytic semigroup. Preprint.

Duncan, T.E., Pasik-Duncan, B. and Stettner, L. (1994a) Almost self-optimizing strategies for the adaptive control of diffusion processes. JOTA, 81, 479-507.

Duncan, T.E., Pasik-Duncan, B. and Stettner, L. (1994b) On discretized MLE in adaptive control of ergodic Markov models. Preprint.

Duncan, T.E., Pasik-Duncan, B. and Stettner, L. (1995) On ergodic control of stochastic evolution equations. Stoch. Anal. Appl., submitted.

Gatarek, D. and Sobczyk, J. (1994) On the existence of optimal controls of Hilbert-valued diffusions. SIAM J. Control Optimiz., 32, 170-175.

Maslowski, B. and Seidler, J. (1993) Ergodic properties of recurrent solutions of stochastic evolution equations. Preprint AVCR MU 81.

Peszat, S. and Zabczyk, J. (1994) Strong Feller property and irreducibility for diffusion on Hilbert spaces. Annals of Prob., to appear.

Stettner, L. (1993) On nearly selfoptimizing strategies for a discrete-time uniformly ergodic adaptive model. JAMO, 27, 161-177. 